\title{
Maintenance and use of cumulative haematology files on a laboratory computer
}

\author{
M. K. AlEXANDER, M. T. CORBETT, AND R. W. REED \\ From Warwick Pathology Laboratory, Lakin Road, Warwick
}

SUMMARY A system is described for the maintenance of cumulative haematology records for selected patients using a laboratory minicomputer with limited storage capacity. Records are indexed by the patient's name and location since, as in the majority of hospital laboratories, there is no unique numbering scheme which covers all patients. The computer system imitates the procedures of manual filing, listing uncertain record matches for human decision.

Applications include the production of a regularly updated printing of cumulative files, the preparation of graphs from cumulative blood count records, and the provision of a summary and follow-up service for general practitioners.

An adequate report filing system is indispensable to the efficient functioning of any clinical laboratory. This is particularly the case in haematology where there are commonly additional clinical responsibilities heavily dependent on accumulated records. There are many ways of keeping records for these purposes: the computer, by virtue of its powers as a storage device and its ability to reproduce the same item of information in different contexts, is one obvious medium whose full potential has yet to be explored.

At the present, there are in hospital practice three main varieties of computer installation which handle laboratory data: an independent laboratory system based usually on a minicomputer and dedicated to the needs of one or more disciplines; a hospital information system which serves a proportion of laboratory functions as one aspect of its overall design; and a variety in which there is a division of functions between a small laboratory computer linked in some way to a larger external system, the latter being used primarily for bulk data storage and retrieval. The merits and limitations of the three approaches are discussed by Cavill et al. (1975). Whatever the undoubted advantages of the two latter methods, it seems clear that, for the present, practical consideration of finance and organisation will restrict the options of many laboratories to the small independent computer, and it is to the problems involved in developing

Received for publication 20 September 1976 filing procedures for such a system in a haematology laboratory that this paper is directed.

The problems are essentially those of the limited capacity of the bulk storage device and the absence of a means of consistently and accurately linking separate records for the same individual. Briefly, the solutions adopted entail the rigorous selection of data to be stored and what is basically a partially computerised simulation of conventional manual filing procedures. (The normal 'look-up' requirement for answering telephoned and similar queries is met by the production of a daily list of all results arranged alphabetically by patient name.)

\section{Equipment}

The haematology laboratory is of medium size, handling in a day about 250 specimens of all kinds, $25 \%$ of which come from general practice. The data processing equipment consists of a Nova 1200 computer with $20 \mathrm{~K}$ core store, twin 2.5 megabyte discs (one of which is exchangeable), a fast paper tape reader and punch, a $135 / \mathrm{lpm}$ line printer, and a graph plotter $(60 \mathrm{~mm} / \mathrm{s})$. The total cost of hardware was approximately $£ 25000$. The manufacturer's standard operating software is used; application programs have been written in Fortran IV.

\section{Data collection}

Programs and daily data files are held on the fixed disc while the exchangeable disc holds the selected 


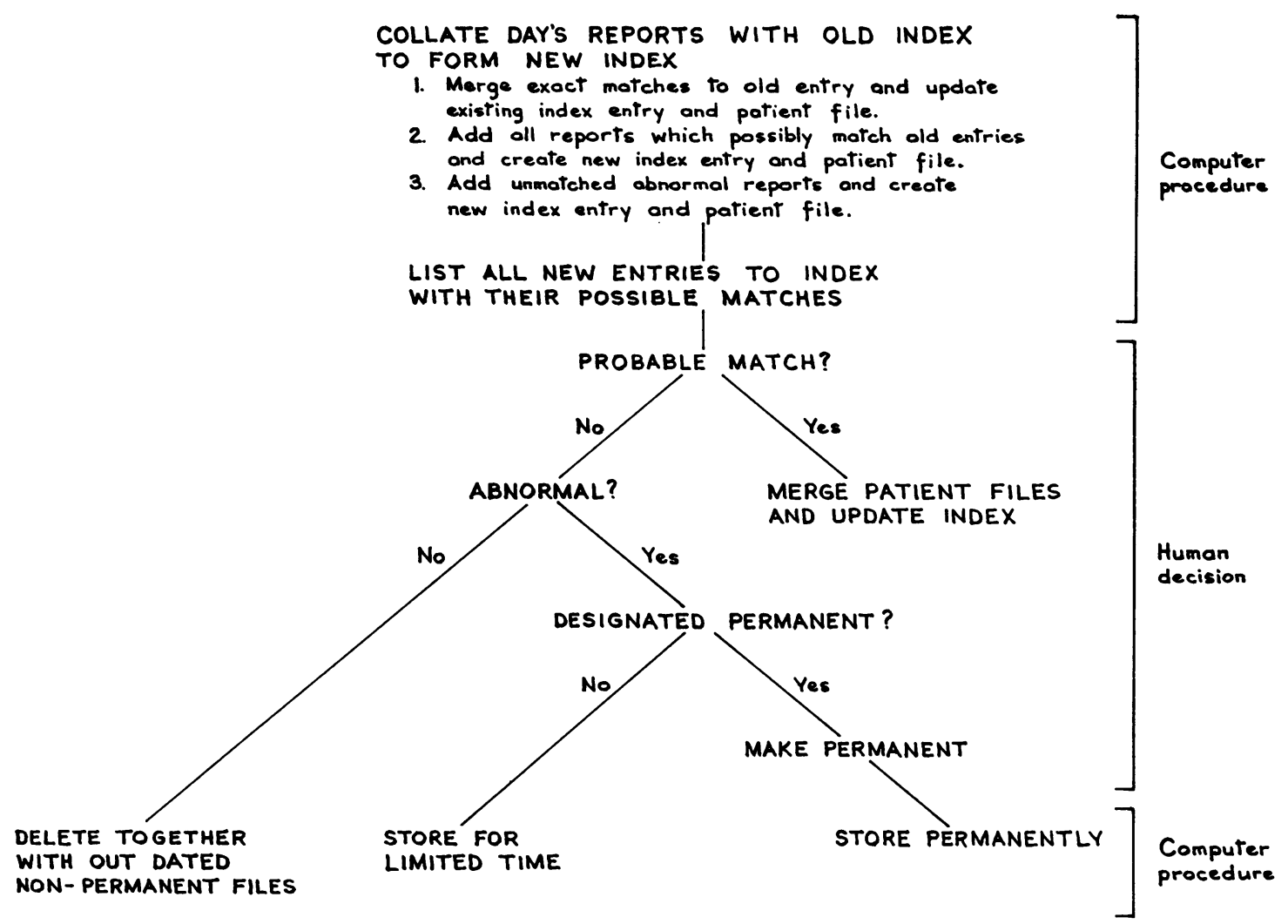

Fig. 1 The filing procedure.

cumulative patient files. The system uses batch processing, patient and test request data, and the results of non-automated tests being prepared offline on punched paper tape. A Coulter Counter Model ' $S$ ' produces results on punched paper tape via an interface to a teletype. Computer-printed worksheets are prepared for the manual recording of differential leucocyte counts, other results, and comments. This information is then punched on to paper tape and entered to the computer for report production, listings, and filing. Reports are produced at intervals throughout the day; listings and filing are carried out once a day.

An analogous system using the same equipment handles the processing of clinical chemistry data, the operations being interleaved with those for haematology.

\section{Data selection}

The reporting run operates on the combined request/ results file to yield two types of output: all records which contain one or more abnormal value (as defined by constants incorporated in the program) are listed separately, the remainder being printed on preprinted report stationery for issue. The 'abnormal' list, amounting to about $15 \%$ of the total, is used by the haematologist for editing before printing of the reports for issue: during this process records are selected for retention in 'permanent' patient files and given a diagnostic code.

The empirical coding system which has been devised uses two levels of classification, the first level being descriptive (eg, 'macrocytic anaemia', 'thrombocytopenia') and the second aetiological. At the same time an 'activity status' is assigned to any file made permanent in a simple fourfold grouping: ' 1 ' indicates that further action is called for from the haematologist, ' 2 ' that action has been requested from the clinician, and ' 3 ' that no specific action is indicated. Category ' 4 ' is used when the patient is known to have died. The codes and activity status form part of the entry on the index of patients' files. 


\section{Filing}

The filing system is based on separate disc files for each individual patient to which later records are added, together with another disc file containing an index to the patient's names and file names.

In summary, the event which leads to the creation of a new patient file is the appearance of an 'abnormal' result in a record which cannot be matched to any existing file. Once a file has been created, any further matching records (normal or abnormal) are added to it. The file once created remains on disc until it has been inactive for 14 days. It is then deleted unless it has in the interim been made permanent.

The filing procedures (Fig. 1) are designed to achieve two main objects: to match new records with existing patient files where appropriate and to assign 'temporary' or 'permanent' attributes to any new patient file created, each procedure being carried out on a partly automatic, partly discretionary basis.

First, all records on the day's report file are assigned distinguishing 'temporary' attributes by the computer according to a set of criteria automatically applied to selected blood count and other results, which divides the entries into broad classes (eg, 'normal', 'macrocytic', 'other abnormality'). The program then goes on to the patient-matching process which is carried out concurrently with the collation and writing of a new patient files index, the basis of comparison being encoded forms of the patients' names derived by means of an algorithm modified from Dolby (1970). The algorithm reduces each name to a single four-character code by a series of rules involving partial elimination and transformation of characters and truncation, thus associating similar names. If a match is found on the 'Dolby' codes of previous and new entries, the program proceeds to a comparison of the full name and ward or general practice code. If an exact match is found, the new record is added to the existing patient file. If no exact match is found, a new patient file is created to contain the new record and a list is printed of patients' names already in the index whose surname 'Dolby' code matches that for one of the names contained in the new record. This procedure allows for the transposition of surname and forename.

New records for which no exact or possible name match is found are treated according to their file attributes: the names of those in the various 'abnormal' categories (together with any others designated by the haematologist) are added to the index, and a new 'temporary' patient file is created, whereas 'normal' records are passed over. The list of possible

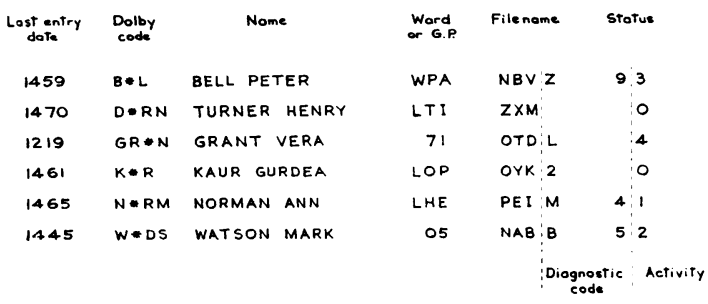

Fig. 2 Structure of the patient files index (date in days from 1 January 1972).

matches is then inspected and decisions are taken as to the probable association of the entries concerned. Merging of entries deemed to be for the same patient is then carried out by a further program, conversational in design, the same program being used to assign 'permanent' attributes, diagnostic codes, and activity status to those files previously nominated by the haematologist at the reporting stage, to correct index entries where necessary, and to delete the remaining 'normal' records (ie, possible matches which are not true matches) and out-dated temporary 'abnormal' records.

'Abnormal' record files which have not been made permanent are retained for at least 14 days and are then deleted unless updated. A file once designated 'permanent' is deleted only at the discretion of the haematologist: before deletion it may, if required, be transferred to paper tape.

The structure of the patient files index (Fig. 2) permits printing of the index alphabetically, by ward or general practice, by diagnostic classification, activity status, or date of last entry.

\section{Applications}

CUMULATIVE LABORATORY RECORDS

Available disc capacity limits the total number of patient files to about 800 , of which 600 are permanent. The latter are culled selectively at regular intervals. The range of test data recorded includes the various elements of the blood count (Coulter values, ESR, reticulocyte, platelet and differential leucocyte counts, together with comment) and the results of B12 and folate assays. Since January 1975 a printed record of all permanent files with alphabetical and nosological indexes has been maintained and updated weekly: this at present serves as the main reference source for previous results on individual patients.

GRAPHIC CUMULATIVE REPORTING

Programs have been developed which enable the graphing by plotter of cumulative blood count data 
from any patient file on disc. Separate disc files containing clinical data, details of treatment, and transfusions are maintained for selected patients, and this material is printed on graphs, which are bound in separate folders for inclusion in the patient's clinical records. On the wards, the graph may be extended and annotated but it is returned to the laboratory at convenient intervals for updating. The selection of patients for inclusion in the service is at the request of the clinician concerned or at the haematologist's discretion. The system has the advantages over manual recording methods of ready duplication, saving of labour when it is decided retrospectively to graph data from a given patient, and uniformity of presentation.

\section{AIDE-MEMOIRES AND SUMMARIES}

More than $25 \%$ of the workload of the laboratory originates from general practice, and a consultative service is provided for over 60 practitioners. The problem of communicating effectively with so many participants is formidable: a partial solution has been achieved by the distribution at fortnightly intervals of summary lists of all abnormal results originating from the different practitioners in the district. The lists include when available the cumulative records of the patients concerned and are annotated with comment and suggestions. They serve both as an aide-mémoire for the practitioner and as an additional channel of communication for the haematologist.

The inclusion in the structure of the file index of 'activity status' enables the generation at regular intervals of summary lists of cases which require the personal attention of the haematologist.

\section{Discussion}

In order to link separate records accumulated for the same individual there must be a method for consistent and accurate identification. In practice, this is an unattainable ideal to which all existing systems are more or less successful approximations. The degree of confidence which can be placed on the linkage varies with the number and reliability of the independent identifiers available, and this in turn determines the type of use to which the information thus associated can be put.

Most large commercial and governmental information systems of necessity demand a high degree of confidence in identification and, as a condition of their functioning, exclude items of data of uncertain provenance. In the hospital field, a few systems of this type are in operation: most entail the generation of individualised computer-readable request documents at initial patient registration (Peters, 1976).
However, the environment in which the average laboratory data-processing system has to operate militates against reliable identification. To give but two examples: emergency hospital admissions (in the absence of round-the-clock registration) and patients referred by general practitioners present difficulties to a system dependent on an otherwise efficient hospital unit numbering scheme, and to this must be added (for less demanding systems) the familiar problems of similarity or misspelling of names and changes of ward or address. Despite all this, it is a fact of everyday experience that even a relatively inefficient laboratory filing system may be valuable provided that the data associated within it are scrutinised critically and used conservatively, the latter term being taken to mean that cumulative records are normally used for the editing and interpretation of fresh results rather than for issue to wards. If used in this way, the effect of the irreducible errors of misfiling is minimised and, for the most part, results only in a relatively unimportant reduction of efficiency due to loss of information.

The choice of an algorithmic method of matching names for possible record linkage was made after experiment with an alternative approach of character-by-character matching, adapted to allow for miskeying of a character or the transposition of two characters (the commonest types of punching error). The latter method, although having certain advantages, proved unacceptably time-consuming as well as failing to associate common variants of names (eg, Dennis and Denis, Graham and Graeme).

When considering the overall efficiency and usefulness of this system of filing, it is necessary to bear in mind, first, its limited objectives, which may conveniently be summarised as 'selective cumulative recording', and, secondly, that, as the resolution of individual filing problems is left largely to human decision, its basic accuracy of record linkage is of the same order as in many conventional laboratory filing systems. In particular, we would emphasise that, although the primary association between records is made with names and locations, in doubtful cases recourse may be had to other discriminants including age, sex, consultant, and registration number when available.

Limiting records filed to those for patients with abnormal findings arising from a single episode of illness-leaving the more general look-up function of laboratory records to a printed daybook type of listing-reduces the volume of material stored in the computer to that small proportion of the total throughput (less than $5 \%$ ) which is in practice required for later comparison. From this point of view the error rate in terms of misfiling or nonfiling has proved to be acceptable. The compactness 
of the cumulative file helps to ensure a proportionately rapid retrieval of the required information.

Professional judgement is not called for in the selection of records for filing to any greater extent than with a purely manual system. The clerical labour required for maintenance of the system averages approximately 30 minutes during the day, while program running times occupy approximately 60 minutes a day.

In practice, although the number of patient files which can be maintained has been dictated by the capacity of the disc, this has not seriously limited the value of the system. Such restrictions as have been experienced relate to the failure to store normal blood counts of patients in whom abnormalities are later found in other types of test, eg, B12 or folate assays, and in the lack of space required to store biochemical results for the patients concerned. The larger capacity disc systems currently becoming available for small computers (eg, 10 Mbyte as opposed to the 5 Mbyte for the system under discussion) will go far to remove these limitations. No significant modification to the programs would be needed to accommodate these facilities; if required they could include the storing of all results for a limited period.

We would suggest that for many haematology laboratories one of the main potential advantages of a minicomputer lies in its ability to support the use of a comparatively simple filing system of this kind.

\section{References}

Cavill, I., Ricketts, C., and Jacobs, A. (1975). Computers in Haematology. Butterworths, London and Boston.

Dolby, J. L. (1970). An algorithm for variable-length proper-name compression. Journal of Library Automation, 3, 257-275.

Peters, M. (1976). Biomedical Computer Technology, 1, 25. 